Ophthalmology. 2013 November ; 120(11): . doi:10.1016/j.ophtha.2013.03.045.

\title{
Evaluation of Ocular Surface Disease in Patients with Glaucoma
}

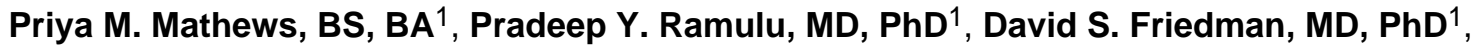 \\ Canan A. Utine, MD, MSc $^{2,3}$, and Esen K. Akpek, MD ${ }^{2}$
}

${ }^{1}$ Division of Glaucoma, The Wilmer Eye Institute, The Johns Hopkins University, Baltimore Maryland ${ }^{2}$ Division of Cornea and External Disease, The Wilmer Eye Institute, The Johns Hopkins University School of Medicine, Baltimore, Maryland ${ }^{3}$ Department of Ophthalmology, Yeditepe University Eye Hospital, Istanbul, Turkey

\begin{abstract}
Purpose-To evaluate the subjective and objective measures of ocular surface disease in patients with glaucoma.
\end{abstract}

Design-Cross-sectional study.

Participants-Sixty-four glaucoma subjects with bilateral visual field (VF) loss and 59 glaucoma suspects with normal VFs.

Methods-Consecutive patients were recruited prospectively from the Wilmer Eye Institute Glaucoma Clinic.

Main Outcome Measures-Tear film breakup time (TBUT), corneal staining score (0-15), and Schirmer's test results were included as objective metrics, whereas the Ocular Surface Disease Index (OSDI) questionnaire was administered to assess symptoms. Total OSDI score, visionrelated subscore (derived from questions about vision and task performance), and discomfortrelated subscore (derived from questions about ocular surface discomfort) were calculated for each subject.

Results-Seventy-five percent (48/64) of glaucoma subjects and 41\% (24/59) of glaucoma suspects were receiving topical medications. The corneal staining grade was greater in glaucoma subjects than in glaucoma suspects ( 6.4 vs. $4.1 ; P<0.001)$, but groups did not differ with regard to TBUT or Schirmer's results ( $P>0.20$ for both). Multivariate regression models showed that topical glaucoma therapy burden was associated with a significantly higher total corneal staining grade ( $\beta$, +0.9 for each additional glaucoma drop; $95 \%$ confidence interval [CI], $0.5-1.3 ; P<0.001$ ), but not with TBUT or Schirmer's results ( $P>0.20$ for both). Glaucoma subjects had significantly higher total OSDI scores than glaucoma suspects ( 16.7 vs. $7.9 ; P<0.001)$. This largely was the result of higher vision-related subscores in the glaucoma group (11.1 vs. $3.3 ; P<0.001)$. Ocular discomfortrelated subscores, however, were similar in both groups (5.7 vs. $4.6 ; P=0.30)$. In multivariate

(C) 2013 by the American Academy of Ophthalmology.

Correspondence: Esen K. Akpek, MD, Ocular Surface Disease and Dry Eye Clinic, Johns Hopkins Jerome L. Greene Sjögren's Syndrome Center, The Wilmer Eye Institute, 600 North Wolfe Street, Maumenee \#317, Baltimore, MD 21287. esakpek@jhmi.edu. Financial Disclosure(s)

The author(s) have made the following disclosure(s): David S. Friedman -Financial support - Alcon, Bausch \& Lomb, Merck, Pfizer, and QLT, Inc. Supported by the National Eye Institute, National Institutes of Health, Bethesda, Maryland (grant no.: EY018595 [P.Y.R.]); a Robert and Helen Schaub Scholar Award from Research to Prevent Blindness, Inc., New York, New York (P.Y.R.); an American Glaucoma Society/Glaucoma Research Foundation Career Development Award (P.Y.R.); a discovery grant from the Jerome L. Greene Sjögren's Syndrome Center (E.K.A.). The sponsors or funding organizations had no role in the design or conduct of this research. 
analyses, each 5-decibel decrement in better-eye VF mean deviation was associated with a 4.7point increase in total OSDI score $(95 \% \mathrm{CI}, 1.9-7.5 ; P=0.001)$ and a 3.7-point increase in the vision-related subscore $(95 \% \mathrm{CI}, 1.7-5.6 ; P<0.001)$ but did not predict a higher discomfort-related subscore $(\beta, 1.1$ point; $P=0.07)$. Topical glaucoma therapy burden was not associated with higher total OSDI score or vision- or discomfort-related subscore $(P>0.20$ for all).

Conclusions-Glaucoma is associated with significant ocular surface disease, and topical glaucoma therapy burden seems predictive of corneal staining severity. However, OSDI is a poor metric for capturing ocular surface disease in glaucoma because symptoms seem to be related largely to VF loss.

Glaucoma is the second leading cause of blindness in the world, affecting more than 60 million people globally. ${ }^{1}$ Although several surgical techniques are available to lower intraocular pressure (IOP), the first line of therapy to treat glaucoma most often is topical eyedrops. ${ }^{2}$ A major concern with topical glaucoma therapy is ocular surface damage because $45 \%$ to $60 \%$ of patients using glaucoma eyedrops are reported to have ocular surface disease $^{3-5}$ Changes in the ocular surface, conjunctiva, and trabecular meshwork are known to occur because of long-term exposure to glaucoma eyedrops ${ }^{6,7}$ and may result from longterm exposure to preservatives, active ingredients, or both. ${ }^{8}$

Currently, no standardized protocol exists for detecting ocular surface disease in glaucoma

patients. ${ }^{9}$ Previous studies generally have adapted the methods of diagnosing ocular surface disease in patients with dry eye syndrome, despite a lack of evidence demonstrating the validity of these tests in glaucoma patients. Subjective symptoms typically are assessed by the Ocular Surface Disease Index (OSDI) questionnaire, and objective clinical tests typically include tear film breakup time (TBUT), conjunctival or corneal staining scores or both, as well as Schirmer's test. ${ }^{10}$

The OSDI questionnaire is used widely and addresses various aspects of ocular surface disease, such as symptoms of ocular discomfort and functional limitations, and yields a single, cumulative score. ${ }^{11}$ However, the multidimensional nature of this questionnaire must be considered when evaluating patients with other coexisting ocular diseases. ${ }^{12}$ For example, glaucoma-related visual field (VF) loss is associated with a lower self-reported quality of life ${ }^{13-15}$ and has been associated with difficulty with several of the specific tasks queried as part of the OSDI, such as reading ${ }^{16,17}$ and driving. ${ }^{18,19}$ In this study, we investigated the association of glaucoma-related VF loss and use of topical glaucoma therapy burden with subjective and objective measures of ocular surface disease. The OSDI responses to questions relating to visual functioning (i.e., reading, computer use, driving) and ocular discomfort (i.e., grittiness, pain) were analyzed separately to determine the types of symptoms reported by glaucoma patients.

\section{Patients and Methods}

The study protocol was approved by the Johns Hopkins University Institutional Review Board. Participants signed written informed consent forms and completed study procedures between July 2009 and April 2011.

\section{Study Subjects}

Consecutive patients 50 years of age or older were recruited prospectively from The Wilmer Eye Institute Glaucoma Clinic. Patients were eligible for enrollment if they reported English fluency and had undergone VF testing at the Glaucoma Clinic within the past 12 months (HFA2; Carl Zeiss Meditec, Inc., Dublin, CA). The VF testing was performed in both eyes over the central $24^{\circ}$ using a size III stimulus and the Swedish interactive threshold algorithm 
standard testing program. Visual field severity was defined by the higher (less negative) mean deviation (MD) between the 2 eyes. Individuals with a history of laser or intraocular injection in the previous week, ocular surgery in the previous 2 months, or visual loss for reasons other than glaucoma were excluded from this study, as described previously. ${ }^{16}$

Two groups of subjects were studied: individuals who were being seen for possible glaucoma (glaucoma suspects) and patients with a known history of glaucoma with documented bilateral VF loss (glaucoma subjects). Grouping was made solely based on the VF results, regardless of glaucoma eyedrop use. Glaucoma suspects had ocular hypertension, or other reasons for suspected glaucoma, and met the following criteria: (1) VF MD better than -3 decibels $(\mathrm{dB})$ in at least 1 eye and better than $-4 \mathrm{~dB}$ in both eyes on the Swedish interactive threshold algorithm standard 24-2 test; (2) glaucoma hemifield test results within normal limits, borderline, or general reduction of sensitivity in both eyes; and (3) presenting visual acuity of 20/40 or better in both eyes on the Early Treatment Diabetic Retinopathy Study chart. Other glaucoma hemifield test results were permitted if the VF test was noted to have low test reliability or excessively high false-positives, although at least 1 eye in each of these 3 subjects had glaucoma hemifield test results of either within normal limits or general reduction of sensitivity. The glaucoma subjects had a known diagnosis of primary open-angle glaucoma, primary angle-closure glaucoma, pseudoexfoliation glaucoma, or pigment dispersion glaucoma and demonstrated: (1) a better-eye visual acuity of at least 20/40, (2) a VF MD worse than $-3 \mathrm{~dB}$ in both eyes, and (3) glaucoma hemifield test results outside normal limits, borderline, or generalized reduction of sensitivity, with the results of at least 1 eye being outside normal limits. For patients with a recent 24-2 VF in only 1 eye and either no recent VF or a 10-2 VF in the second eye, the better-eye MD was taken from the recent 24-2 VF. Detailed description of the study groups is presented elsewhere. ${ }^{16}$

\section{Ocular Surface Disease Index Questionnaire}

The OSDI questionnaire was administered to all participants by a masked ophthalmic technician. Individual OSDI questions were scored on a 4-point Likert scale, with scores of $0,1,2,3$, and 4 corresponding to answers of none of the time, some of the time, half of the time, most of the time, and all of the time, respectively. A total score was calculated using the following equation: $12.5 \times$ [(sum of individual question scores)/(number of questions answered)], yielding a total score ranging from ranging from 0 to 100 . Total scores were categorized for severity as follows: normal $=0$ to 12 , mild $=13$ to 22 , moderate $=23$ to 32 , and severe $=33$ to $100.10,11,20$

In addition to the total OSDI score, the same formula was used to compute 2 subscores: (1) OSDI vision-related score and (2) OSDI ocular discomfort-related score. The OSDI visionrelated score corresponded to the 6 questions (nos. 4 through 9) that assessed the effect of ocular surface disease on visual functioning (blurred vision, poor vision, and limitations with reading, driving, computer use, and watching television). The OSDI ocular discomfort score corresponded to the 6 questions (nos. 1 through 3 and 10 through 12) evaluating symptoms relating to irritation or discomfort (light sensitivity; grittiness or pain/soreness; and discomfort in windy, dry, or air-conditioned environments). Subscale scores ranged from 0 to 50 .

\section{Assessment of Ocular Surface Grading}

Ocular surface staining grading was performed by 1 of 2 masked graders (E.K.A. or P.Y.R.). The tests were administered in the order listed here. The TBUT was measured as time between the last complete blink and first appearance of a growing micelle after $5 \mu \mathrm{l}$ of anesthetic-free, preservative-free $2 \%$ sodium fluorescein was placed in the inferior bulbar 
conjunctiva. Three TBUT measurements were obtained for each eye 1 minute after instilling the eyedrop (with a maximum value of 10 seconds) using the cobalt blue light of a slit lamp and a Wratten 12 yellow filter and were averaged for each eye.

Corneal staining was evaluated using the National Eye Institute grading system. Within 2 minutes after TBUT testing, the extent of punctate epithelial erosions in 5 regions (superior, inferior, temporal, nasal, and central) of the cornea was graded on a 4-point scale (0-3) using the cobalt blue light of a slit lamp and Wratten 12 yellow filter paper. ${ }^{21}$ The scores of the 5 regions were summed to obtain a total corneal staining grade for each eye, ranging from 0 to 15. Finally, the Schirmer's test was performed without anesthesia in each eye at least 5 minutes after corneal staining assessment and results were obtained at 5 minutes. ${ }^{22}$

\section{Ocular Medication History}

Participants reported current glaucoma eyedrops used along with the number of drops placed per day in each eye. The total amount of benzalkonium chloride (BAK) per day was calculated from the number of drops per day and the BAK concentration for each specific agent obtained from the Food and Drug Administration medication insert ${ }^{23}$ or manufacturing company, based on an estimated drop size of $50 \mu \mathrm{l}$.

\section{Measurement of Vision and Covariates}

Presenting visual acuity was assessed binocularly and was converted into negative logarithm of the minimum angle of resolution units. ${ }^{24}$ Contrast sensitivity was measured as the number of letters read correctly on the Pelli-Robson chart and was transformed to a logarithmic scale. ${ }^{25}$ The presence of significant lenticular changes was assessed for each eye after pupillary dilation, as described previously. ${ }^{26}$

Chart review and questionnaires were used to collect information about age, race or ethnicity, education, and history of glaucoma surgery. Comorbid illnesses likely to affect functionality significantly were determined and summed for all subjects. Depressive symptoms were assessed using part D of the General Health Questionnaire. ${ }^{27}$ The MiniMental State Examination was used to evaluate cognitive ability. ${ }^{28}$ The total number of dry eye-associated systemic medications used was identified based on patient-provided medication lists and published literature describing the classes of dry eye-associated medications. ${ }^{29}$

\section{Statistical Methods and Programming}

Group differences were analyzed using the Student $t$ test for continuous variables and the chi-square test for categorical variables. Predictors of continuous OSDI scores and subscores and ocular surface metrics were analyzed as bivariate (age-adjusted) and multivariate linear regression models. Robust regression models were used when model residuals failed the Shapiro-Wilk normality test. Predictors of being in a higher OSDI severity class (none, mild, moderate, or severe) were identified using ordinal logistic regression models, and the proportional odds assumption was confirmed by the Brant test. The primary study outcomes were the association of glaucoma status, glaucoma severity, burden of topical glaucoma medication, or a combination of (1) total OSDI score, (2) OSDI subscores, and (3) ocular surface measures.

Glaucoma medication burden was measured primarily as the maximum number of glaucoma eyedrops used daily in either or each eye at the time of enrollment. Additional confirmatory analyses were performed in which medication burden was evaluated as (1) bottles of glaucoma medication used, (2) glaucoma medication agents used (accounting for combination medications), (3) daily drop applications, and (4) amount of BAK per day for 
either eye. Covariates included in multivariate regression models were (1) age, race, and sex; (2) variables associated with total OSDI score or any ocular surface measure in ageadjusted analyses; and (3) relevant variables identified in previous research studies. ${ }^{29}$ Data analysis was performed with STATA version 12 (STATA Corp., College Station, TX) and figures were produced by R version 2.15.1 (R Development Core Team, Vienna, Austria).

\section{Results}

One hundred twenty-three individuals (64 glaucoma subjects and 59 glaucoma suspects) completed study procedures and were included for analysis. Patient demographics are summarized in Table 1. Patients with glaucoma were older than glaucoma suspects (71.6 vs. 67.0 years; $P=0.004$ ) but were not significantly different in terms of sex, race, education, employment status, cognitive ability, depressive symptoms, comorbid illnesses, or systemic medications ( $P>0.20$ for all). Glaucoma patients had greater VF loss, worse visual acuity, and lower contrast sensitivity compared with the glaucoma suspects $(P<0.001)$. Patients with glaucoma also used a greater number of glaucoma eyedrops per day (median, 2 ; range, $0-8$ ) than glaucoma suspects (median, 0 ; range, $0-4 ; P<0.001$ ).

Glaucoma subjects had higher average total OSDI scores $(16.7$ vs. $7.9 ; P<0.001)$ and visionrelated subscores ( 11.1 vs. $3.3 ; P<0.001)$ compared with glaucoma suspects but did not differ in mean ocular discomfort-related subscore (5.7 vs. 4.6; $P=0.30$; Table 2, Fig 1). Frequencies of OSDI-based disease classification (mild, moderate, severe) also differed by glaucoma status $(P<0.001 ;$ Fig 2$)$. Patients with glaucoma had a higher total corneal staining grade than the glaucoma suspects (right eye: 6.4 vs. $4.1, P<0.001$; left eye: 6.3 vs. $4.2, P=$ $0.003)$ but did not differ with regard to average TBUT or Schirmer's test outcomes $(P>0.20$ for all; Table 2). Significant group differences also were found in each of the 5 corneal staining zones $(P<0.05$ for all). Glaucoma eyedrop burden and severity of corneal staining both increased with glaucoma severity up through moderate disease severity and then decreased slightly in individuals with the most severe glaucoma (Figs 3 and 4).

\section{Predictors of Ocular Surface Disease Index Scores: Multivariate Analysis}

In multivariate linear regression models, greater better-eye VF loss was associated with a higher total OSDI score ( +4.7 per 5-dB decrement in MD; 95\% confidence interval [CI], $1.9-7.5 ; P=0.001)$ and a higher vision-related subscore $(+3.7$ per $5-\mathrm{dB}$ decrement in $\mathrm{MD}$; $95 \% \mathrm{CI}, 1.7-5.6 ; P<0.001)$ but was not associated significantly with a higher ocular discomfort-related subscore $(+1.1$ units per $5-\mathrm{dB}$ decrement in MD; 95\% CI, 0.1-2.4; $P=$ 0.07; Table 3). Higher total and vision-related scores $(P<0.05)$, but not discomfort-related subscores $(P=0.7)$, also were found with worse better-eye visual acuity. The presence of additional comorbid illness also was associated with a higher total OSDI score and higher vision-related subscore ( $P<0.05$ for both) but not with ocular discomfort-related subscore, although the difference did not reach statistical significance $(P=0.27)$. No other tested variables, including maximum number of glaucoma eyedrops per day and total BAK dose per day, were associated with total OSDI score or either subscore $(P>0.20$ for all).

A greater likelihood of being in a worse OSDI category (normal, mild, moderate, or severe) was found with greater better-eye VF loss (odds ratio, 1.7 per 5-dB decrement in MD; 95\% CI, $1.3-2.4 ; P=0.001$ ) and worse visual acuity (odds ratio, 1.5 per 0.1 -logarithm of the minimum angle of resolution increment; 95\% CI, 1.0-2.3; $P=0.03$ ), whereas the number of glaucoma drops used per day was not associated with a greater likelihood of a worse OSDI score category (odds ratio, 1.0 per 1-drop increase; $95 \%$ CI, $0.8-1.2 ; P=0.85$; Table 4). Participants who were female or had a comorbid illness also were more likely to be in a worse OSDI score category $(P<0.05)$, whereas no association was found with age, sex, previous glaucoma surgery, or systemic medications. 


\section{Predictors of Ocular Surface Clinical Tests: Multivariate Analysis}

The maximum number of glaucoma drops per day used in either eye was associated with a higher corneal staining grade (+0.9 per 1-drop increment; 95\% CI, 0.5-1.3; $P<0.001)$, but not with average TBUT ( +0.0 seconds per 1-drop increase; 95\% CI, -0.4 to $0.5 ; P=0.95$ ) or Schirmer's test score ( $+0.4 \mathrm{~mm}$ wetting per 1-drop increase; $95 \% \mathrm{CI},-0.7$ to $1.5 ; P=0.48$; Table 5). Similar results were observed with all other metrics of glaucoma eyedrop load (right eye and left eye maximum number of bottles, agents, daily drop applications, and amount of BAK per day). Additionally, men were more likely to have a higher average TBUT than women $(+1.8$ seconds; $95 \% \mathrm{CI}, 0.5-3.1 ; P=0.007)$. No other tested variables, including glaucoma status, were associated with the average TBUT, corneal staining grade total, or Schirmer's test scores ( $P>0.05$ for all).

\section{Discussion}

Our results suggest that OSDI score changes in patients with glaucoma primarily reflect greater difficulty with vision and vision-related tasks as a result of glaucoma-related vision loss. Severity of VF loss itself was a strong predictor of higher OSDI score and OSDI-based disease category, and this effect was driven primarily by an increase in functional disability rather than ocular discomfort. Additionally, the burden of topical IOP-lowering medication was associated strongly with the extent of corneal punctate epithelial erosions but was not associated with tear film abnormality or tear production. These findings suggest that glaucoma medications do indeed damage the ocular surface but may not produce greater discomfort symptoms in patients on an established treatment regimen.

Many research studies have used the OSDI questionnaire to estimate the prevalence of ocular surface disease in the glaucoma population. Here, our findings suggest that this instrument may not be appropriate for this purpose because combining all responses into a single total score may hide differences between the various aspects of the disease, which only become clear when subscores are evaluated separately. Indeed, a recent review of the literature ${ }^{9}$ acknowledged the lack of evidence establishing the OSDI as a valid measure of ocular surface disease in glaucoma patients. The authors considered several potential confounders of using OSDI in this context, including glaucoma disease itself, natural fluctuation in symptoms depending on the time of day or environment, and altered perception of symptoms resulting from a decrease in corneal sensitivity from glaucoma therapy. By separating the OSDI score into subscores pertaining to either functionality or discomfort symptoms, we demonstrated the multidimensionality of the OSDI questionnaire that actually has been reported previously. ${ }^{12}$ These results suggest that higher OSDI scores in glaucoma largely are the result of overlapping visual symptoms and functional difficulties between glaucoma and ocular surface disease and not because of ocular discomfort symptoms introduced by glaucoma therapy. Our findings differed from those of Skalicky et $\mathrm{al},{ }^{15}$ who found that both functional difficulties related to VF loss and BAK dose predicted higher OSDI score. However, the authors did not analyze the 2 components of the OSDI score (visual function versus ocular discomfort related symptoms) separately, making it unclear if symptoms of discomfort were indeed related to medication use. Additionally, patients using greater amounts of topical IOP-lowering medications are likely to have worse VF loss, potentially contributing to the observed association between BAK load and total OSDI score.

Several previous studies have demonstrated that glaucoma patients have difficulty with the visual functions queried as part of the OSDI. For example, OSDI questions about reading and computer work are likely to be affected in glaucoma given prior evidence that reading difficulties are common in glaucoma and that greater VF loss results in decreased reading speed, reading fatigue, and more frequent reading impairment. ${ }^{13,16,17,30}$ Likewise, the OSDI 
question about night driving is likely to be affected in glaucoma given prior evidence of driving restriction and self-reported driving difficulty among subjects with glaucoma. ${ }^{18,19}$ Finally, difficulty with poor or blurry vision is to be expected in glaucoma, particularly as VF loss and decline in contrast sensitivity become more pronounced. Rossi et al ${ }^{31}$ also found that among patients with glaucoma, the presence of objective ocular surface disease further negatively influenced glaucoma-related quality of life, although these findings may reflect the fact that surface disease was more common in subjects with more advanced VF loss resulting from greater glaucoma treatment burden.

Our study confirms the previously reported association between use of glaucoma eyedrops and corneal staining, suggesting that medications, medication preservatives such as BAK, or both are damaging directly to the ocular surface. ${ }^{4,32}$ However, our findings differ from studies using other objective methods to assess the ocular surface, such as those that found an association between topical IOP-lowering medication use and tear film measurements. ${ }^{33,34}$ Discrepancies among objective tests have been attributed to limitations such as variance in measurements and scoring, heterogeneous populations, and environmental differences. ${ }^{9}$ Additionally, we did not observe a difference in ocular discomfort symptoms between glaucoma subjects and glaucoma suspects. One unifying hypothesis is that IOP-lowering medication use may lead to punctate epithelial erosions and a subsequent decrease in corneal sensitivity, therefore masking symptoms and explaining the similar prevalence of ocular discomfort-related symptoms. ${ }^{35,36}$ Our study also confirms the discordance between patient-reported symptoms and clinical evaluations when detecting ocular surface disease. ${ }^{33,37}$ A similar explanation has been invoked for other conditions such as diabetic retinopathy, where a decrease in corneal sensitivity most likely contributes to the lack of ocular discomfort symptoms. ${ }^{38}$

Although glaucoma patients may be less symptomatic in terms of ocular surface disease, clinicians should assess the ocular surface proactively because of ongoing inflammation and should consider treatment to prevent subsequent damage that may lead to conditions such as chronic conjunctivitis and corneal haze. Additionally, ocular surface disease and damage to the epithelium resulting from topical glaucoma treatment potentially may lead to the failure of future surgical interventions, including trabeculectomies. ${ }^{39}$

There are several other notable findings in our study. The proportion of abnormal total OSDI scores among our study subjects was similar to that of previous studies; however, our results indicate that this is an inaccurate estimate of the prevalence of ocular surface disease among the glaucoma population. ${ }^{4,32,40}$ The presence of comorbid disease potentially affecting functionality also was associated with the OSDI total score and the OSDI vision-related subscore, providing further evidence that the patient's overall health condition must be considered when interpreting the OSDI score. We showed that multiple forms of measuring glaucoma topical therapy burden (including maximum number of glaucoma bottles, applications per day, drops per day, and total amount of BAK) had very similar trends of association with the outcomes studied, suggesting that any of these metrics can be used in clinical practice. Additionally, we observed that women were more likely to be in a worse OSDI-based disease category and to have a shorter TBUT, consistent with previous studies showing a higher prevalence of ocular surface disease among women. ${ }^{3,41}$

Our study has several limitations. The VF tests were performed up to 12 months before the other variables were measured (i.e., topical IOP-lowering medication use) and could have changed during this interval. However, previous studies have reported rates of VF loss in glaucoma patients under routine care to be less than $0.5 \mathrm{~dB}$ per year. ${ }^{42,43}$ Also, a substantial proportion of the glaucoma suspects were using topical IOP-lowering medications, potentially influencing subjective symptoms and ocular surface metrics. We believe this 
perceived limitation actually is a major strength of the study because it allowed us to assess the independent effect of glaucoma therapy burden across a spectrum of VF loss. Another limitation of our study is that we did not collect information regarding the duration of glaucoma therapy, which may be associated with the development of ocular surface disease. Additionally, our study design included glaucoma subjects who underwent surgical intervention and may have discontinued glaucoma therapy afterward. These patients, most of whom were in the severe disease category, may explain the slight decrease in average number of eye drops and total corneal staining grade compared with the moderate disease category (Tables 3 and 4). Finally, it is possible, or even likely, that ocular discomfort symptoms resulting from topical IOP-lowering therapy were not observed in our study because symptomatic patients were transferred to other medications that produce fewer symptoms or were offered laser or surgical treatment to obviate the need for medical therapy. Indeed, the true symptoms and ocular surface disease associated with topical IOPlowering medicines are difficult to estimate because patients intolerant of medications are likely to change therapy, achieve therapy by surgical means, or simply choose to be noncompliant with therapy to minimize ocular surface discomfort.

In summary, our findings suggest that the OSDI questionnaire is not a valid assessment of ocular surface disease in the glaucoma population because changes in total OSDI score are driven by changes in visual function associated with greater vision loss. Clinical tests such as ocular surface staining scores are more likely a better method of diagnosing ocular surface disease in patients with glaucoma. Ocular surface staining should be performed routinely to minimize epithelial damage, therefore minimizing the risk of failure for future glaucoma interventions as well. Further studies should focus on developing and using better subjective and objective tools for evaluating ocular surface disease in glaucoma patients.

\section{References}

1. Quigley HA, Broman AT. The number of people with glaucoma worldwide in 2010 and 2020 . Br J Ophthalmol. 2006; 90:262-7. [PubMed: 16488940]

2. Ramulu PY, Corcoran KJ, Corcoran SL, Robin AL. Utilization of various glaucoma surgeries and procedures in Medicare beneficiaries from 1995 to 2004. Ophthalmology. 2007; 114:2265-70. [PubMed: 17466376]

3. Erb C, Gast U, Schremmer D. German register for glaucoma patients with dry eye. I. Basic outcome with respect to dry eye. Graefes Arch Clin Exp Ophthalmol. 2008; 246:1593-601. [PubMed: 18648841]

4. Leung EW, Medeiros FA, Weinreb RN. Prevalence of ocular surface disease in glaucoma patients. J Glaucoma. 2008; 17:350-5. [PubMed: 18703943]

5. Garcia-Feijoo J, Sampaolesi JR. A multicenter evaluation of ocular surface disease prevalence in patients with glaucoma. Clin Ophthalmol. 2012; 6:441-6. [PubMed: 22536034]

6. Pisella PJ, Pouliquen P, Baudouin C. Prevalence of ocular symptoms and signs with preserved and preservative free glaucoma medication. Br J Ophthalmol. 2002; 86:418-23. [PubMed: 11914211]

7. Baudouin C. The ocular surface in glaucoma. Cornea. 2009; 28(suppl):S14-9.

8. Baudouin C, Hamard P, Liang H, et al. Conjunctival epithelial cell expression of interleukins and inflammatory markers in glaucoma patients treated over the long term. Ophthalmology. 2004; 111:2186-92. [PubMed: 15582072]

9. Pflugfelder SC, Baudouin C. Challenges in the clinical measurement of ocular surface disease in glaucoma patients. Clin Ophthalmol. 2011; 5:1575-83. [PubMed: 22125404]

10. Schiffman RM, Christianson MD, Jacobsen G, et al. Reliability and validity of the ocular surface disease index. Arch Ophthalmol. 2000; 118:615-21. [PubMed: 10815152]

11. Walt, J. Ocular Surface Disease Index (OSDI) Administration and Scoring Manual. Irvine, CA: Allergan, Inc; 2004. 
12. Dougherty BE, Nichols JJ, Nichols KK. Rasch analysis of the ocular surface disease index (OSDI). Invest Ophthalmol Vis Sci. 2011; 52:8630-5. [PubMed: 21948646]

13. Nelson P, Aspinall P, Papasouliotis O, et al. Quality of life in glaucoma and its relationship with visual function. J Glaucoma. 2003; 12:139-50. [PubMed: 12671469]

14. Goldberg I, Clement CI, Chiang TH, et al. Assessing quality of life in patients with glaucoma using the Glaucoma Quality of Life-15 (GQL-15) questionnaire. J Glaucoma. 2009; 18:61-2.

15. Skalicky SE, Goldberg I, McCluskey P. Ocular surface disease and quality of life in patients with glaucoma. Am J Ophthalmol. 2012; 153:1-9. [PubMed: 21872203]

16. Ramulu PY, Swenor BK, Jefferys JL, et al. Difficulty with out-loud and silent reading in glaucoma. Invest Ophthalmol Vis Sci. 2013; 54:666-72. [PubMed: 23074207]

17. Ramulu PY, West SK, Munoz B, et al. Glaucoma and reading speed: the Salisbury Eye Evaluation Project. Arch Ophthalmol. 2009; 127:82-7. [PubMed: 19139345]

18. Ramulu PY, West SK, Munoz B, et al. Driving cessation and driving limitation in glaucoma: the Salisbury Eye Evaluation Project. Ophthalmology. 2009; 116:1846-53. [PubMed: 19592110]

19. Ramulu PY. Glaucoma and disability: which tasks are affected, and at what stage of disease? Curr Opin Ophthalmol. 2009; 20:92-8. [PubMed: 19240541]

20. Miller KL, Walt JG, Mink DR, et al. Minimal clinically important difference for the Ocular Surface Disease Index. Arch Ophthalmol. 2010; 128:94-101. [PubMed: 20065224]

21. Dursun D, Ertan A, Bilezikci B, et al. Ocular surface changes in keratoconjunctivitis sicca with silicone punctum occlusion. Curr Eye Res. 2003; 26:263-9. [PubMed: 12854053]

22. Liew M, Zhang M, Kim E, Akpek EK. Prevalence and predictors of Sjögren's syndrome in a prospective cohort of patients with aqueous-deficient dry eye. Br J Ophthalmol. 2012; 96:1498503. [PubMed: 23001257]

23. [Accessed March 23, 2013] DailyMed: Current Medication Information. Available at: http:// dailymed.nlm.nih.gov/dailymed/about.cfm

24. Bailey IL, Bullimore MA, Raasch TW, Taylor HR. Clinical grading and the effects of scaling. Invest Ophthalmol Vis Sci. 1991; 32:422-32. [PubMed: 1993595]

25. Elliott DB, Bullimore MA, Bailey IL. Improving the reliability of the Pelli-Robson contrast sensitivity test. Clin Vis Sci. 1991; 6:471-5.

26. Ramulu PY, Maul E, Hochberg C, et al. Real-world assessment of physical activity in glaucoma using an accelerometer. Ophthalmology. 2012; 119:1159-66. [PubMed: 22386950]

27. Goldberg DP, Hillier VF. A scaled version of the General Health Questionnaire. Psychol Med. 1979; 9:139-45. [PubMed: 424481]

28. Folstein MF, Folstein SE, McHugh PR. "Mini-Mental State": a practical method for grading the cognitive state of patients for the clinician. J Psychiatr Res. 1975; 12:189-98. [PubMed: 1202204]

29. Schein O, Hochberg MC, Munos B, et al. Dry eye and dry mouth in the elderly: a population-based assessment. Arch Intern Med. 1999; 159:1359-63. [PubMed: 10386512]

30. Nelson P, Aspinall P, O'Brien C. Patients' perception of visual impairment in glaucoma: a pilot study. Br J Ophthalmol. 1999; 83:546-52. [PubMed: 10216052]

31. Rossi GC, Tinelli C, Pasinetti GM, et al. Dry-eye syndrome-related quality of life in glaucoma patients. Eur J Ophthalmol. 2009; 19:572-9. [PubMed: 19551671]

32. Valente C, Iester M, Corsi E, Rolando M. Symptoms and signs of tear film dysfunction in glaucomatous patients. J Ocul Pharmacol Ther. 2011; 27:281-5. [PubMed: 21557633]

33. Ghosh S, O'Hare F, Lamoureux E, et al. Prevalence of signs and symptoms of ocular surface disease in individuals treated and not treated with glaucoma medication. Clin Experiment Ophthalmol. 2012; 40:675-81. [PubMed: 22394358]

34. Labbe A, Terry O, Brasnu E, et al. Tear film osmolarity in patients treated for glaucoma or ocular hypertension. Cornea. 2012; 31:994-9. [PubMed: 22710490]

35. Martone G, Frezzotti P, Tosi GM, et al. An in vivo confocal microscopy analysis of effects of topical antiglaucoma therapy with preservative on corneal innervation and morphology. Am J Ophthalmol. 2009; 147:725-35. [PubMed: 19181302] 
36. Van Went C, Alalwani H, Brasnu E, et al. Corneal sensitivity in patients treated medically for glaucoma or ocular hypertension [in French]. J Fr Ophtalmol. 2011; 34:684-90. [PubMed: 22093372]

37. Nichols KK, Nichols JJ, Mitchell GL. The lack of association between signs and symptoms in patients with dry eye disease. Cornea. 2004; 23:762-70. [PubMed: 15502475]

38. Dogru M, Katakami C, Inoue M. Tear function and ocular surface changes in noninsulin-dependent diabetes mellitus. Ophthalmology. 2001; 108:586-92. [PubMed: 11237914]

39. Mirza SK, Higginbotham EJ. Iatrogenic glaucoma therapy failure: the adverse effects of topical antiglaucoma medication treatment outcome. Expert Rev Clin Pharmacol. 2009; 2:87-99.

40. Fechtner RD, Godfrey DG, Budenz D, et al. Prevalence of ocular surface complaints in patients with glaucoma using topical intraocular pressure-lowering medications. Cornea. 2010; 29:618-21. [PubMed: 20386433]

41. Portello JK, Rosenfield M, Bababekova Y, et al. Computer-related visual symptoms in office workers. Ophthalmic Physiol Opt. 2012; 32:375-82. [PubMed: 22775070]

42. De Moraes CG, Juthani VJ, Liebmann JM, et al. Risk factors for visual field progression in treated glaucoma. Arch Ophthalmol. 2010; 129:562-8. [PubMed: 21555607]

43. Kwon YH, Kim CS, Zimmerman MB, et al. Rate of visual field loss and long-term visual outcome in primary open-angle glaucoma. Am J Ophthalmol. 2001; 132:47-56. [PubMed: 11438053] 


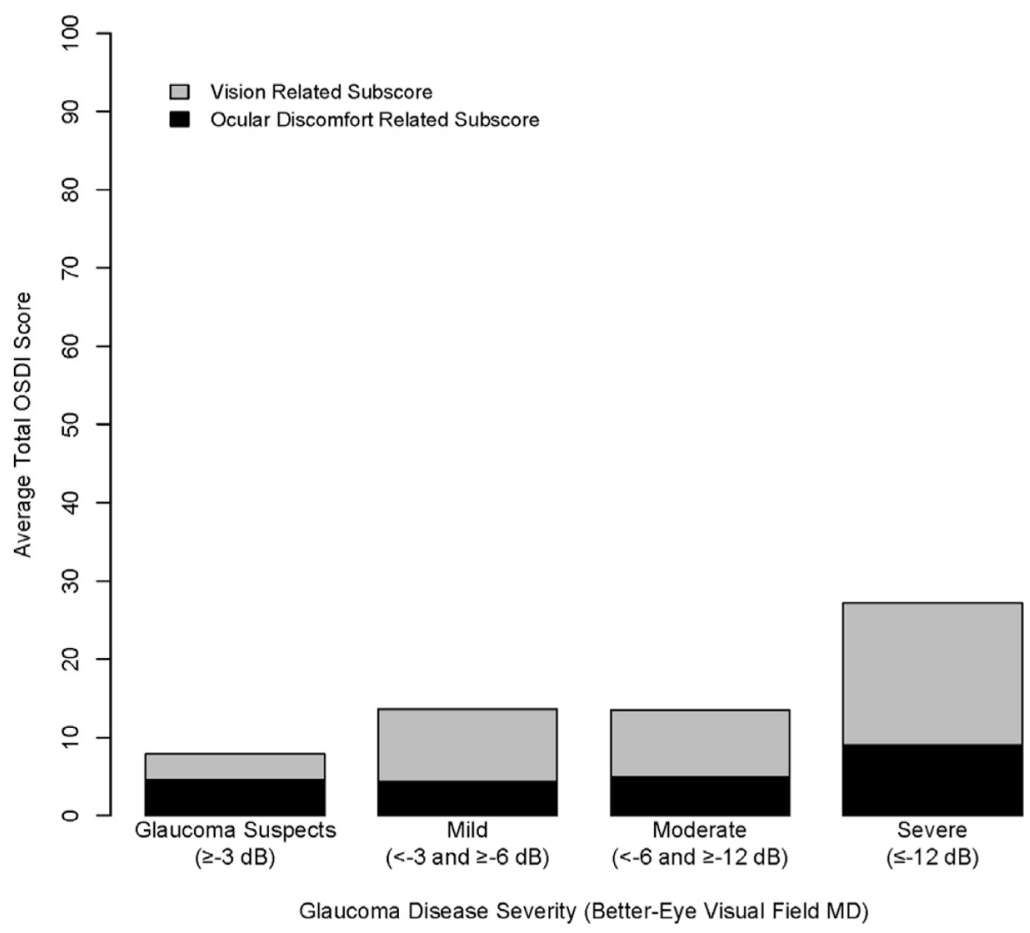

Figure 1.

Bar graph showing average Ocular Surface Disease Index (OSDI) scores according to glaucoma disease severity based on severity of visual field loss. Total OSDI score is equal to the sum of the vision-related and ocular discomfort-related subscores (each subscore range, $0-50)$. Scores shown are averages for each category of visual field loss. $\mathrm{dB}=$ decibels; $\mathrm{MD}$ $=$ mean deviation. 


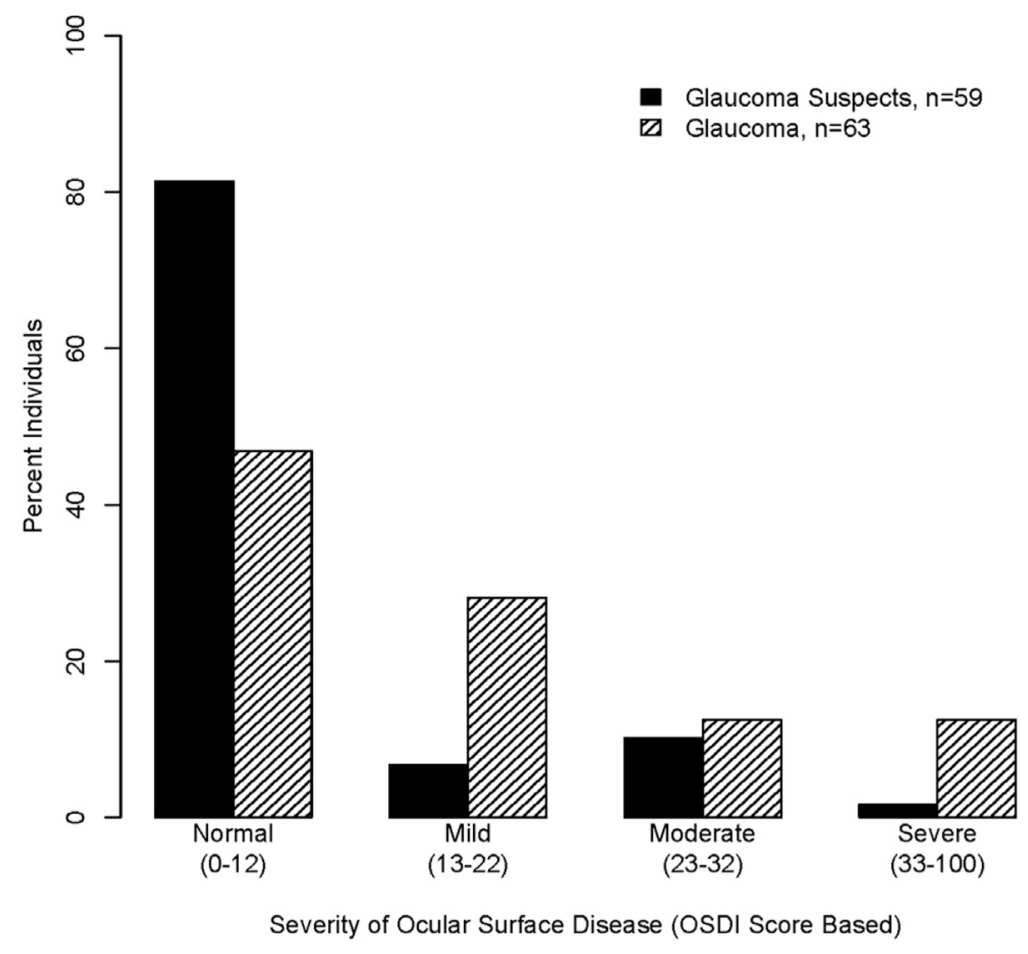

Figure 2.

Bar graph showing the severity of ocular surface disease based on total Ocular Surface Disease Index (OSDI) score according to glaucoma status. Individuals were categorized based on OSDI score. Percentages shown are proportions within the glaucoma or glaucoma suspect group. 


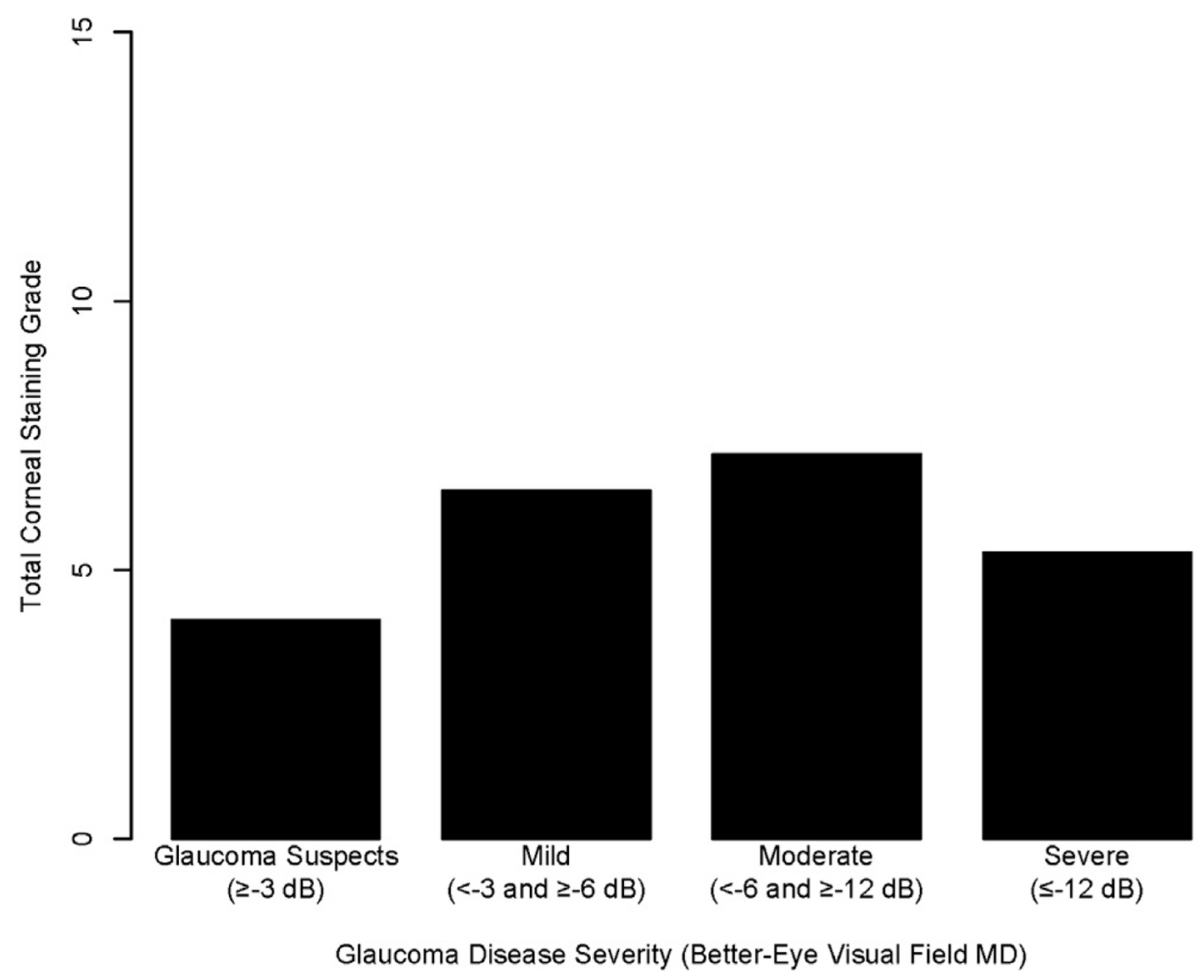

Figure 3.

Bar graph showing the total corneal staining grade according to glaucoma disease severity based on visual field loss. Corneal staining grade in 5 regions (superior, inferior, temporal, nasal, and central cornea) were summed to obtain a total value (using the National Eye Institute scale range, 0-15). Average total corneal staining grade is shown for each category of better-eye visual field loss. $\mathrm{dB}=$ decibels; $\mathrm{MD}=$ mean deviation. 


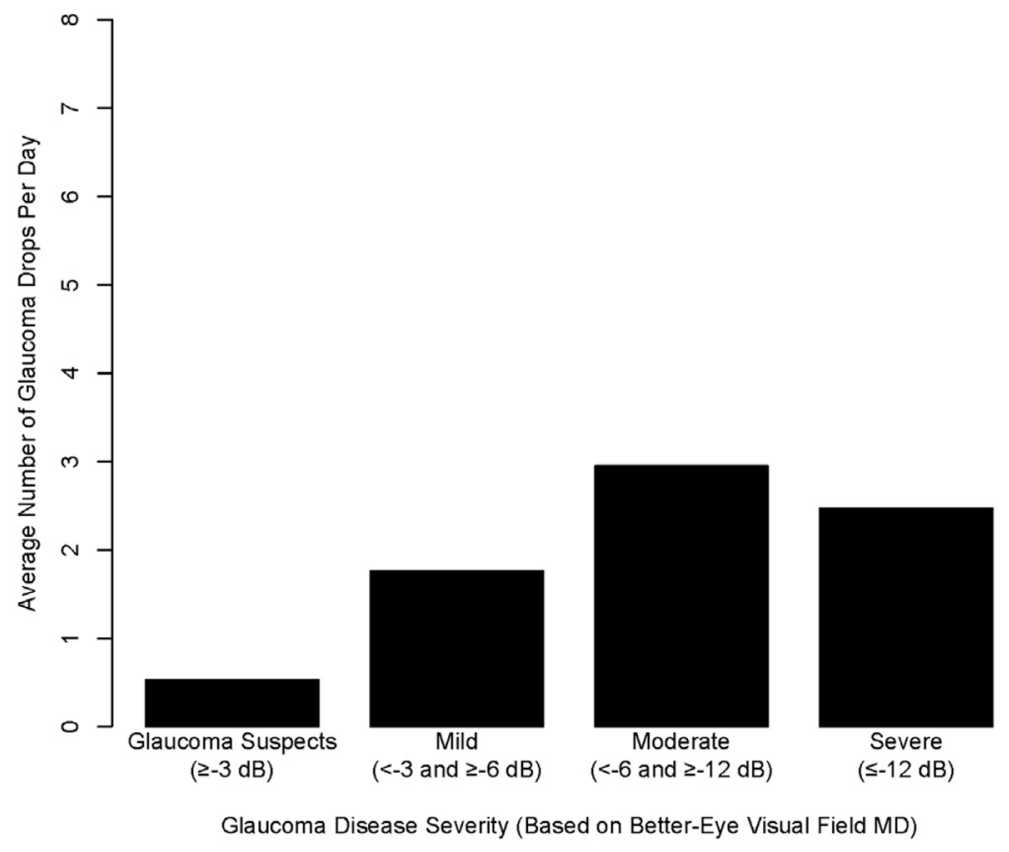

Figure 4.

Bar graph showing the average number of glaucoma drops per day according to glaucoma disease severity based on visual field loss. Maximum number of glaucoma drops (in either or each eye) was averaged for each category of better-eye visual field loss. $\mathrm{dB}=$ decibels; $\mathrm{MD}=$ mean deviation . 
Table 1

Characteristics of Study Participants by Glaucoma Status

\begin{tabular}{|c|c|c|c|}
\hline & Glaucoma Suspects $(n=64)$ & Glaucoma Subjects $(n=59)$ & $P$ Value \\
\hline \multicolumn{4}{|l|}{ Demographics } \\
\hline Age $(\mathrm{yrs})^{*}$ & 71.6 & 67.0 & $<0.01$ \\
\hline Male sex $(\%)$ & 42 & 37 & 0.58 \\
\hline African-American race $(\%)$ & 20 & 18 & 0.82 \\
\hline Education (yrs) & 15.2 & 15.4 & 0.59 \\
\hline Employed (\%) & 42 & 47 & 0.56 \\
\hline \multicolumn{4}{|l|}{ Vision } \\
\hline Visual field MD (better eye) ${ }^{*}$ & -8.9 & 0.2 & $<0.001$ \\
\hline Binocular acuity $(\log M A R) *$ & 0.09 & 0.00 & $<0.001$ \\
\hline Binocular $\log \mathrm{CS}^{*}$ & 1.67 & 1.93 & $<0.001$ \\
\hline Significant cataract/PCO $(\%)^{\dagger}$ & 11 & 7 & 0.42 \\
\hline Glaucoma surgery $(\%)^{\dagger}$ & 63 & 2 & $<0.001$ \\
\hline \multicolumn{4}{|l|}{ Glaucoma therapy } \\
\hline Currently using glaucoma eyedrops (\%) & 75 & 41 & $<0.001$ \\
\hline Maximum number of drops/day, median (range) & $2(0-8)$ & $0(0-4)$ & $<0.001$ \\
\hline Total amount of BAK per day, $\mu \mathrm{g}$ & 15.9 & 6.7 & $<0.001$ \\
\hline \multicolumn{4}{|l|}{ Health } \\
\hline MMSE score & 27.4 & 27.7 & 0.21 \\
\hline Depressive symptoms (\%) & 8 & 7 & 0.83 \\
\hline Comorbid illnesses (\%) & 28 & 27 & 0.91 \\
\hline Systemic medications ${ }^{*}$ & 4.0 & 3.8 & 0.68 \\
\hline \multicolumn{4}{|c|}{$\begin{array}{l}\mathrm{BAK}=\text { benzalkonium chloride } \mathrm{CS}=\text { contrast sensitivity } ; \log \mathrm{MAR}=\log \text { arithm of the minimum angle of resolution; } \mathrm{MD}=\text { mean deviation; MMSE } \\
=\text { Mini-Mental State Examination } \mathrm{PCO}=\text { posterior capsular opacification. }\end{array}$} \\
\hline \multicolumn{4}{|c|}{ Indicates median values. Mean values shown for all other continuous variables. } \\
\hline t In 1 or both eyes. & & & \\
\hline
\end{tabular}

Ophthalmology. Author manuscript; available in PMC 2014 November 01. 
Table 2

Outcomes of Ocular Surface Disease Index Scores and Objective Dry Eye Findings by Glaucoma Status

\begin{tabular}{|c|c|c|c|}
\hline & Glaucoma Subjects $(n=64)$ & Glaucoma Suspects $(n=59)$ & $P$ Value \\
\hline \multicolumn{4}{|l|}{ Ocular Surface Disease Index } \\
\hline Average total score $*$ & 16.7 & 7.9 & $<0.001$ \\
\hline Ocular discomfort related ${ }^{\dagger}$ & 5.7 & 4.6 & 0.30 \\
\hline Vision related ${ }^{t}$ & 11.1 & 3.3 & $<0.001$ \\
\hline \multicolumn{4}{|c|}{ Ocular surface measurements (mean/median) } \\
\hline \multicolumn{4}{|c|}{ Tear film breakup time (seconds) } \\
\hline Right eye & $4.2 / 3.0$ & $4.4 / 3.0$ & 0.75 \\
\hline Left eye & $4.5 / 3.3$ & $4.6 / 3.3$ & 0.91 \\
\hline \multicolumn{4}{|c|}{ Total corneal staining score $(0-15)$} \\
\hline Right eye & $6.4 / 7.0$ & $4.1 / 3.0$ & $<0.001$ \\
\hline Left eye & $6.3 / 7.0$ & $4.2 / 3.0$ & 0.003 \\
\hline \multicolumn{4}{|l|}{ Schirmer's test (mm) } \\
\hline Right eye & $13.1 / 10.0$ & $15.3 / 11.0$ & 0.27 \\
\hline Left eye & $12.9 / 10.0$ & $12.7 / 10.0$ & 0.93 \\
\hline \multicolumn{4}{|c|}{ Ocular Surface Disease Index total score (range, 0-100). } \\
\hline \multicolumn{4}{|c|}{ Score pertaining to 6 ocular discomfort-related questions (range, $0-50$ ). } \\
\hline
\end{tabular}


Table 3

Predictors of Ocular Surface Disease Severity Measured by Ocular Surface Disease Index Scores, Multivariate Analysis

\begin{tabular}{|c|c|c|c|c|}
\hline \multirow[b]{2}{*}{ Variable } & \multirow[b]{2}{*}{ Interval } & \multicolumn{3}{|c|}{ Ocular Surface Disease Index Score, $\beta$ ( $95 \%$ Confidence Interval) } \\
\hline & & Total Score ${ }^{*}$ & Vision-Related Score ${ }^{\dagger}$ & Ocular Discomfort Score \\
\hline \multicolumn{5}{|l|}{ Therapy } \\
\hline Glaucoma eyedrops per day & Maximum no., either eye & $-0.7(-2.1$ to 0.8$)$ & $-0.7(-1.6$ to 0.3$)$ & $-0.1(-0.8$ to 0.7$)$ \\
\hline \multicolumn{5}{|l|}{ Glaucoma severity } \\
\hline Visual acuity, better eye & $0.1 \log$ MAR worse & $2.1(0.1-4.1)^{\xi}$ & $2.0(0.4-3.5) / /$ & $0.2(-0.7$ to 1.1$)$ \\
\hline VF mean deviation, better eye & $5 \mathrm{~dB}$ worse & $4.7(1.9-7.5) / /$ & $3.7(1.7-5.6) / /$ & $1.1(0.1-2.4)$ \\
\hline Previous glaucoma surgery & Vs. no glaucoma surgery & $-0.5(-5.3$ to 4.3$)$ & $0.1(-3.7$ to 3.9$)$ & $-0.7(-2.9$ to 1.6$)$ \\
\hline \multicolumn{5}{|l|}{ Demographics } \\
\hline Age & 10 Years older & $-2.3(-5.9$ to 2.5$)$ & $-1.4(-3.9$ to 1.0$)$ & $-0.9(-2.5$ to 0.7$)$ \\
\hline Male & Vs. female & $-2.3(-7.2$ to 1.7$)$ & $-0.9(-4.1$ to 2.3$)$ & $-1.6(-3.9$ to 0.8$)$ \\
\hline African American & Vs. not African American & $0.5(-5.5$ to 6.5$)$ & $-0.4(-3.6$ to 4.3$)$ & $0.1(-2.8$ to 3.0$)$ \\
\hline Comorbid illness & $\geq 1$ Illness & $5.5(0.2-10.8)^{\xi}$ & $4.2(0.2-8.2)^{\xi}$ & $1.3(-1.0$ to 3.5$)$ \\
\hline Systemic medications ${ }^{I}$ & No. of medication classes & $0.03(-1.7$ to 1.8$)$ & $-0.1(-1.2$ to 1.1$)$ & $0.1(-0.8$ to 0.9$)$ \\
\hline \multicolumn{5}{|c|}{$\mathrm{dB}=$ decibels $; \log \mathrm{MAR}=$ logarithm of the minimum angle or resolution; $\mathrm{VF}=$ visual field. } \\
\hline \multicolumn{5}{|c|}{ Bolded values indicate statistically significant findings at $P<0.05$. } \\
\hline \multicolumn{5}{|c|}{ *cular Surface Disease Index total score (range, 0-100). } \\
\hline \multicolumn{5}{|c|}{ Score pertaining to 6 ocular discomfort-related questions (range, $0-50$ ). } \\
\hline \multicolumn{5}{|c|}{${ }^{*}$ Score pertaining to 6 vision-related questions (range, $0-50$ ). } \\
\hline \multicolumn{5}{|l|}{$\xi_{P<0.05}$} \\
\hline$/ 1 / P<0.01$ & & & & \\
\hline
\end{tabular}


Table 4

Predictors of Having a Worse Level of Ocular Surface Disease Severity as Judged by Ocular Surface Disease Index Category, Multivariate Analysis

\begin{tabular}{|c|c|c|}
\hline Variable & Interval & Likelihood of Worse OSDI Score* Odds Ratio (95\% Confidence Interval) \\
\hline \multicolumn{3}{|l|}{ Glaucoma therapy } \\
\hline Glaucoma eyedrops per day & Maximum no., either eye & $0.98(0.77-1.24)$ \\
\hline \multicolumn{3}{|l|}{ Glaucoma severity } \\
\hline Visual acuity, better eye & $0.1 \log$ MAR worse & $1.54(1.04-2.28)^{\dagger}$ \\
\hline VF mean deviation, better eye & $5 \mathrm{~dB}$ worse & $1.74(1.25-2.42)^{*}$ \\
\hline Previous glaucoma surgery & Vs. no glaucoma surgery & $1.35(0.56-3.28)$ \\
\hline \multicolumn{3}{|l|}{ Demographics } \\
\hline Age & 10 years older & $0.82(0.50-1.35)$ \\
\hline Male & Vs. female & $0.36(0.15-0.86)^{\dagger}$ \\
\hline African American & Vs. not African American & $1.06(0.41-2.76)$ \\
\hline \multicolumn{3}{|l|}{ Health } \\
\hline Comorbid illness & $\geq 1$ Illness & $2.55(1.03-6.32)^{\dagger}$ \\
\hline Systemic medications $\xi$ & No. of medication classes & $0.90(0.69-1.18)$ \\
\hline \multicolumn{3}{|c|}{$\mathrm{dB}=$ decibels $; \log \mathrm{MAR}=$ logarithm of the minimum angle or resolution; OSDI $=$ Ocular Surface Disease Index; VF $=$ visual field } \\
\hline \multicolumn{3}{|c|}{ Bolded values indicate statistically significant findings at $P<0.05$. } \\
\hline \multirow{2}{*}{\multicolumn{3}{|c|}{$\begin{array}{l}\text { * Worse score being a higher category of disease based on OSDI classification (normal vs. mild vs. moderate vs. severe). Odds ratios taken from } \\
\text { multivariate model including visual acuity, visual field mean deviation, age, sex, race, comorbidities affecting reading, previous glaucoma surgery } \\
\text { and systemic medications as covariates. }\end{array}$}} \\
\hline & & \\
\hline \multicolumn{3}{|l|}{${ }^{\dagger} P<0.05$} \\
\hline \multicolumn{3}{|l|}{${ }^{*} P<0.01$} \\
\hline
\end{tabular}


Table 5

Association of Objective Dry Eye Findings with Glaucoma Medication Therapy and Disease Severity, Multivariate Analysis

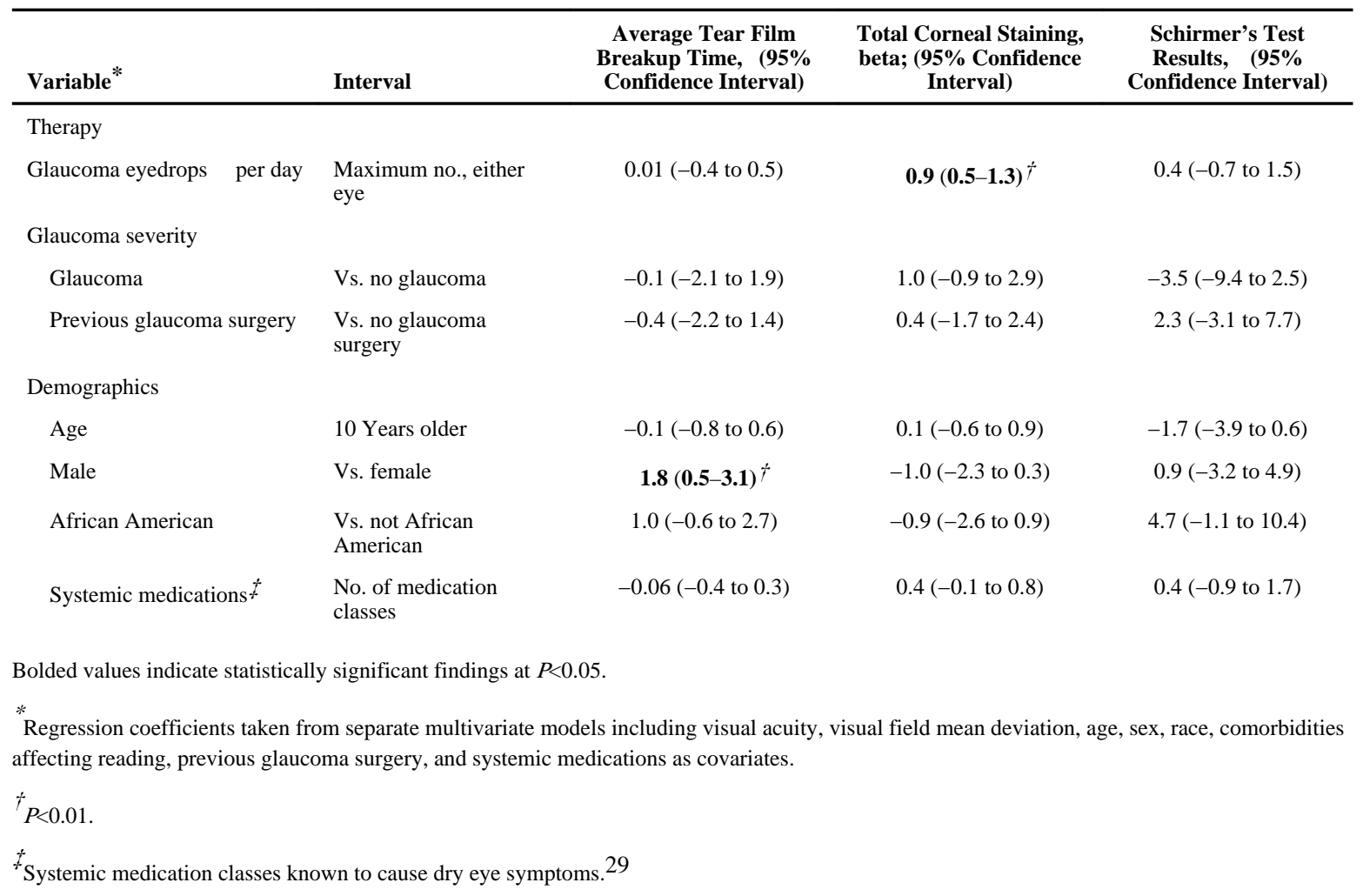

